QMUL-PH-01-10

hep-th/0110034

\title{
Fivebranes Wrapped on SLAG Three-Cycles and Related Geometry
}

\author{
Jerome P. Gauntlett*1 ${ }^{* 1}$ Nakwoo Kim ${ }^{\dagger 2}$, Dario Martelli*3 and Daniel Waldram*4 \\ * Department of Physics \\ Queen Mary \& Westfield College \\ University of London \\ Mile End Rd, London E1 $4 \mathrm{NS}, \mathrm{UK}$ \\ ${ }^{\dagger}$ Max-Planck-Institut für Gravitationsphysik \\ Albert-Einstein-Institut \\ Am Mühlenberg 1, D-14476 Golm, Germany
}

\begin{abstract}
We construct ten-dimensional supergravity solutions corresponding to the near horizon limit of IIB fivebranes wrapping special Lagrangian three-cycles of constant curvature. The case of branes wrapping a three-sphere provides a gravity dual of pure $\mathcal{N}=2$ super-Yang-Mills theory in $D=3$. The non-trivial part of the solutions are seven manifolds that admit two $G_{2}$ structures each of which is covariantly constant with respect to a different connection with torsion. We derive a formula for the generalised calibration for this general class of solutions. We discuss analogous aspects of the geometry that arises when fivebranes wrap other supersymmetric cycles which lead to $\operatorname{Spin}(7)$ and $S U(N)$ structures. In some cases there are two covariantly constant structures and in others one.
\end{abstract}

${ }^{1}$ E-mail: j.p.gauntlett@qmw.ac.uk

2 E-mail: kim@aei-potsdam.mpg.de

${ }^{3}$ E-mail: d.martelli@qmw.ac.uk

${ }^{4}$ E-mail: d.j.waldram@qmw.ac.uk 


\section{Introduction}

The near horizon limit of supergravity solutions corresponding to branes wrapping supersymmetric cycles provide gravity duals to the field theories arising on the branes. By exploiting the fact that the field theories are "twisted" [四], the corresponding supergravity solutions can be constructed first in an appropriate gauged supergravity and then uplifted to $D=10$ or $D=11$. This was first demonstrated in [2] and has been generalised to a number of different cases in [3] - [17].

An interesting class of examples to study is type IIB NS- or D-fivebranes wrapping supersymmetric cycles, since one can obtain supersymmetric Yang-Mills (SYM) theory on the unwrapped part of the fivebrane in the IR. $\mathcal{N}=1 \mathrm{SYM}$ in $D=4$ was studied in [3], $\mathcal{N}=1 \mathrm{SYM}$ in $D=3$ in [5, 10, 11] and $\mathcal{N}=2 \mathrm{SYM}$ in $D=4$ in 14, 15. One of the purposes of this paper is to extend these investigations to $\mathcal{N}=2$ SYM in $D=3$. To do so we consider fivebranes wrapping special Lagrangian (SLAG) three-cycles in Calabi-Yau threefolds. As discussed in [2] the appropriate limit to decouple gravity implies that only the local geometry of the SLAG three-cycle is important.

If we wrap the fivebranes on a SLAG three-sphere, for which the deformed conifold is the appropriate local model of the geometry without the backreaction, we obtain pure $\mathcal{N}=2 \mathrm{SYM}$ in $D=3$. Perturbatively this theory has a Coulomb branch but a superpotential is generated by instanton (monopole) effects [18, 19, 20] and there is no stable vacuum. As such it might seem impossible to obtain a gravity dual. However, these instanton effects are suppressed in the large $N$ limit and might not be expected to survive in the supergravity approximation. This was observed in the context of $\mathcal{N}=2 \mathrm{SYM}$ in $D=4$ in [14], where the supergravity solutions incorporated all perturbative effects but not the non-perturbative corrections. Indeed we will construct singular supergravity solutions and by probing these solutions with a fivebrane argue that they describe a slice of the perturbative Coulomb branch of $\mathcal{N}=2 \mathrm{SYM}$ in $D=3$.

If a Chern-Simons term with suitable co-efficient is added to pure $\mathcal{N}=2 \mathrm{SYM}$ in $D=3$, one expects that there is a unique confining ground state 21]. Following [3, 10, 11] one then anticipates that a regular supergravity solution dual to these theories should exist, which should include non-zero NS flux on the SLAG threesphere to account for the Chern-Simons term [5]. The present work can be viewed as a first step toward the construction of these solutions in the same way that the work of [5] led to [10, 11] (who used [22]) for the confining $\mathcal{N}=1 D=3$ theories.

The non-trivial part of the $D=10$ solutions constructed here are seven-dimensional manifolds with non-vanishing NS three-form $H$ and dilaton. Defining two connections with totally anti-symmetric torsion $\nabla^{ \pm}=\nabla \pm \frac{1}{2} H$, where $\nabla$ is the Levi-Civita connection, we show that the holonomy of each of these connections is in $G_{2}$. In particular we show that the seven manifolds admit two $G_{2}$ structures, specified by associative three forms, one of which is covariantly constant with respect to $\nabla^{+}$and the other with respect to $\nabla^{-}$. We also show that this is a general result for type IIB (and type IIA) backgrounds preserving this amount of supersymmetry. In addition we present the appropriate notion of generalised calibration. In particular we derive 
an expression for the dual six-form potential (which has a seven-form field strength that is dual to the three form $H$ ) in terms of either of the $G_{2}$ structures and the dilaton.

A similar phenomenon was observed in [14] for fivebranes wrapping a two-cycle in Calabi-Yau two-folds. For this case the non-trivial part of the geometry was six-dimensional. It was shown that the manifold admits two commuting complex structures and one of these is covariantly constant with respect to $\nabla^{+}$and the other with respect to $\nabla^{-}$. Moreover, the dual six-form potential can be constructed from either of these complex structures and the dilaton. By contrast a different kind of six dimensional geometry arises when fivebranes wrap a two-cycle inside a CalabiYau threefold. It was shown in [23] that the geometry found in [2] admits just one complex structure that is covariantly constant with respect to one of the connections with torsion. The dual six-form potential can still be constructed from this complex structure and the dilaton. The reason for the difference between the resulting two kinds of geometry in these two cases is that in the former case there are overall transverse directions (two) to the fivebrane wrapping the two-cycle, whereas in the latter case, there are none. This means that after incorporating the back-reaction of the fivebrane on the geometry, in the former case the non-trivial manifold jumps from four dimensions, the Calabi-Yau two-fold, to six as in the solution [14, 15], whereas in the latter example it starts at six, the Calabi-Yau threefold, and remains at six, in the full solution [2].

We show that this holds more generally. For example, when fivebranes wrap SLAG three-cycles there is one overall transverse direction and the corresponding solutions are seven-dimensional with two $G_{2}$-structures. By contrast when fivebranes wrap associative three-cycles in manifolds with $G_{2}$ holonomy there are no overall transverse directions and the corresponding non-trivial seven manifold [5, 10, 11] has a single $G_{2}$ structure. We also discuss other cases that include manifolds with $S U(N)$ and $\operatorname{Spin}(7)$ structures and derive the expression for the generalised calibrations.

Note: In the process of writing up this paper, we became aware of [24]. The results of that paper have some overlap with sections 2, 3, 5 and 6 of the work presented here.

\section{NS fivebranes wrapped on a SLAG three-sphere}

To obtain an $\mathcal{N}=2$ super-Yang-Mills theory in three dimensions, we consider type IIB fivebranes wrapped on a special Lagrangian three-cycle in a Calabi-Yau threefold. We start by recalling that in the limit that the string coupling is set to zero, keeping the string scale fixed, a configuration of $N$ IIB NS fivebranes is described by the six-dimensional IIB little string theory [25]. This theory flows in the IR to $D=6$ super-Yang-Mills theory. Further, it preserves sixteen supercharges transforming as $\left(\mathbf{4}_{+}, \mathbf{2}_{+}\right)+\left(\mathbf{4}_{-}, \mathbf{2}_{-}\right)$under $S O(1,5) \times S O(4)_{R}$ where $S O(4)_{R}$ is the group of $R$ symmetries. Geometrically this group describes rotations in directions normal to the brane. In the large $N$ limit, the theory has a gravity dual, given by the near horizon 
limit of $N$ NS-fivebranes, namely,

$$
\begin{aligned}
\mathrm{d} s^{2} & =\mathrm{d} \xi_{1,5}^{2}+N\left(\mathrm{~d} \rho^{2}+\mathrm{d} \Omega_{3}^{2}\right), \\
\mathrm{e}^{-2 \Phi} & =\mathrm{e}^{-2 \Phi_{0}} \mathrm{e}^{2 \rho}
\end{aligned}
$$

where $\mathrm{d} \Omega_{3}^{2}$ is the metric on a three-sphere, $\mathrm{d} \xi_{1,5}^{2}$ is the Minkowski metric on $\mathbb{R}^{1,5}$ and we have set $\alpha^{\prime}=1$. There is also a NS three-form flux through the three-sphere, normalised so that the integral of the three-form $H / 4 \pi^{2}$ over the sphere is $N$.

To obtain pure $\mathcal{N}=2$ Yang-Mills theory in $D=3$ in the IR we consider this little string theory compactified on $\mathbb{R}^{1,2} \times S^{3}$. The $S O(1,5)$ Lorentz group of the fivebrane is then broken to $S O(1,2) \times S O(3)$. Naively, such a compactification breaks all the supersymmetries. However, by considering a twisted theory four supercharges can be preserved. To do this, we split the $R$-symmetry $S O(4)_{R} \rightarrow S O(3)_{R}$ and then identify the $S O(3)$ spin connection of the three-sphere with $S O(3)_{R}$. Geometrically this is exactly the same twisting that arises in the local description of a fivebrane wrapping a special Lagrangian three-cycle. In this setting the $S O(3)$ part of the $R$-symmetry corresponds to the symmetry group of the normal bundle to the fivebrane inside the Calabi-Yau threefold.

It is straightforward to see that this twisting indeed preserves $\mathcal{N}=2$ supersymmetry in $D=3$. First note that the preserved supersymmetries of the fivebrane transform as two copies of $(\mathbf{2}, \mathbf{2}, \mathbf{2})$ under $S O(1,2) \times S O(3) \times S O(3)_{R}$. After twisting these transform as two copies of $(\mathbf{2}, \mathbf{1})+(\mathbf{2}, \mathbf{3})$ under $S O(1,2) \times S O(3)_{D}$, where $S O(3)_{D}$ is the diagonal subgroup of the two $S O(3)$ factors. It is then the singlets of $S O(3)_{D}$ which are the preserved supersymmetries. The four scalars of a single fivebrane transform as a 4 of $S O(4)_{R}$ and hence as a triplet and a singlet of $S O(3)_{R}$. Geometrically, this simply corresponds to the split of the four-dimensional space transverse to the branes into three directions within the Calabi-Yau threefold and one remaining flat direction. The non-trivial twisting of the normal directions means that the triplet of scalars really describe a section of the normal bundle to the SLAG three-cycle within the Calabi-Yau threefold. Given the identification of $S O(3)_{R}$ with the $S O(3)$ rotations in the tangent space of the cycle, they can also be viewed as one-forms, that is, sections of the cotangent bundle of the three-cycle. This matches the standard result that the normal deformations of a SLAG three-cycle are given by harmonic one-forms on the three-cycle [26]. As there are no harmonic one-forms on a three-sphere, it is necessarily a rigid SLAG three-cycle within a Calabi-Yau threefold and the four scalar fields give rise to one real massless scalar in $D=3$ coming from the singlet. The gauge fields on the fivebrane also have no scalar zero-modes on the three-sphere and thus simply give rise to a $D=3$ gauge field. These fields plus the fermionic partners comprise the field content of pure $D=3 \mathcal{N}=2 U(1)$ Yang-Mills theory. Generalising to $N$ fivebranes gives rise to $S U(N)$ gauge group.

Note that we could also consider wrapping on other constant curvature cycles. In particular, we would get the same theory in the IR if we considered fivebranes wrapping a lens space, $S^{3} / \Gamma$. Alternatively, if we wrapped on a torus $T^{3}$, we would get three additional chiral adjoint matter multiplets from zero modes of the scalar triplet and scalar zero modes of the gauge fields. In this case, there are actually 16 
preserved supercharges, the matter and gauge fields form a single multiplet and the gauge theory in the IR is simply the dimensional reduction of $D=6$ super-YangMills theory. Finally, we could also consider the fivebranes wrapping a compact space constructed as a quotient of hyperbolic space, $H^{3} / \Gamma$. In this case, we again get additional $\mathcal{N}=2$ chiral matter multiplets in the adjoint representation. We will briefly mention the structure of the supergravity solutions for $H^{3}$ in the next section, but will otherwise concentrate on the $S^{3}$ case.

\section{Supergravity solution}

We first note that the IIB supergravity solutions describing wrapped NS fivebranes have only NS-NS fields non-vanishing and hence are also solutions of $\mathcal{N}=1$ supergravity in $D=10$. To explicitly construct the near horizon limits, we follow the strategy of [2] and first construct the solutions within a suitable gauged supergravity and then uplift to $D=10$. The relevant $D=7$ gauged supergravity is obtained from the consistent truncation of $\mathcal{N}=1$ supergravity in $D=10$ on a three-sphere. This gives the $D=7 S O(4)$ gauged supergravity of [27] with the gauge fields arising from the isometries of the three-sphere. A reader not interested in the methods used to derive the solution, could skip the first subsection and begin directly with the full solution in ten dimensions. We also note that given this solution the corresponding solution for wrapped D-fivebranes can then be simply obtained by S-duality.

\subsection{Supergravity solution in $\mathrm{D}=7$ gauged supergravity}

Starting from the bosonic Lagrangian of the $D=7 S O(4)$ gauged supergravity, let us derive BPS equations for the wrapped branes using the effective Lagrangian method given in [14]. One can also check that the same BPS equations are obtained directly from the supersymmetry variations as given in [27, and that solutions of the BPS equations preserve $1 / 8$ of the supersymmetry. The bosonic field content of the gauged

supergravity [27] (see also [28]) comprises of a metric, gauge fields $F^{a b}$ in the adjoint of $S O(4)$, a three-form and ten scalar fields in a symmetric four-by-four matrix $T_{i j}$.

Geometrically the gauge fields describe the twisting of the normal bundle to the fivebrane. Thus to incorporate the twisting discussed in the last section we need to have non-vanishing gauge fields in an $S O(3)$ subgroup of $S O(4)$. We realise this by defining $F^{a} \equiv \frac{1}{2} \epsilon^{a b c} F^{b c}$ for $a, b, c=1,2,3$ and set $F^{a 4}=0$. An ansatz for the scalar fields consistent with the twisting, preserving $S O(3) \subset S O(4)$ is given by

$$
T_{i j}=\mathrm{e}^{y / 4} \operatorname{diag}\left(\mathrm{e}^{x}, \mathrm{e}^{x}, \mathrm{e}^{x}, \mathrm{e}^{-3 x}\right) .
$$

Finally, we set the seven dimensional three-form to zero. It is important to check that such an ansatz is consistent with the three-form, scalar and metric equations of motion, as is easily verified in this case.

With this ansatz the equations of motion are encoded in the following seven 
dimensional Lagrangian

$$
\begin{array}{r}
\mathcal{L}=\sqrt{g}\left\{R-\frac{5}{16} \partial_{\mu} y \partial^{\mu} y\right. \\
-3 \partial_{\mu} x \partial^{\mu} x-\frac{1}{4} \mathrm{e}^{-y / 2-2 x} F_{\mu \nu}^{a} F^{a \mu \nu} \\
\left.+\frac{1}{2} g^{2} \mathrm{e}^{y / 2}\left(3 \mathrm{e}^{2 x}-\mathrm{e}^{-6 x}+6 \mathrm{e}^{-2 x}\right)\right\}
\end{array}
$$

which can be derived from [27] or [28]. For the metric and gauge fields we use the ansatz

$$
\begin{aligned}
\mathrm{d} s^{2} & =\mathrm{e}^{2 f(r)}\left(\mathrm{d} \xi^{2}+\mathrm{d} r^{2}\right)+\frac{a^{2}(r)}{4}\left(\sigma_{1}^{2}+\sigma_{2}^{2}+\sigma_{3}^{2}\right) \\
A^{a} & =\frac{1}{2 g} \sigma^{a}
\end{aligned}
$$

where $\mathrm{d} \xi^{2}$ is the Minkowski metric on $\mathbb{R}^{1,2}$, and the left-invariant one-forms of $S U(2)$ satisfying $\mathrm{d} \sigma^{a}=\frac{1}{2} \epsilon^{a b c} \sigma^{b} \wedge \sigma^{c}$ give the round metric on $S^{3}$. In particular note that we have explicitly incorporated the required twisting by setting the $S O(3)$ gauge fields equal to the $S O(3)$ spin connection of the three-sphere.

When we uplift to $D=10$, in order to find solutions corresponding to wrapped NS-fivebranes, we require that the string-frame warp factor of the $\mathrm{d} \xi^{2}$ term is unity. This implies that, as in [14], we set $y=-4 f$. Substituting this ansatz into the Lagrangian (3) we obtain a one-dimensional effective action which in terms of the new variables

$$
\mathrm{e}^{2 A}=\mathrm{e}^{2 f} a^{3}, \quad \mathrm{e}^{2 h}=\mathrm{e}^{-2 f} a^{2},
$$

is written as

$$
\begin{aligned}
L & =\mathrm{e}^{2 A}\left[4 \dot{A}^{2}-3 \dot{h}^{2}-3 \dot{x}^{2}-V\right] \\
V(h, x) & =-6 \mathrm{e}^{-2 h}+\frac{3}{2 g^{2}} \mathrm{e}^{-4 h-2 x}-\frac{g^{2}}{2}\left(3 \mathrm{e}^{2 x}+6 \mathrm{e}^{-2 x}-\mathrm{e}^{-6 x}\right) .
\end{aligned}
$$

The equations of motion derived from this Lagrangian, combined with the constraint of setting the Hamiltonian to zero (arising from diffeomorphisms in the radial variable) give the differential equations satisfied by $A, h$, and $x$. This system can then be reduced to a set of first-order Hamiltonian equations together with an associated Hamilton-Jacobi equation. Choosing $F=\mathrm{e}^{2 A} W(h, x)$ as a principle function, the Hamilton-Jacobi equation gives rise [14] to the following non-linear equation for the superpotential $W$

$$
V=\frac{1}{4}\left(\frac{1}{3} \partial_{h} W^{2}+\frac{1}{3} \partial_{x} W^{2}-W^{2}\right) .
$$

By inspection, one finds the solution $W=-g\left(\mathrm{e}^{-3 x}+3 \mathrm{e}^{x}+3 g^{-2} \mathrm{e}^{-2 h-x}\right)$, from which 
the first-order BPS equations then follow, and read

$$
\begin{aligned}
& \dot{A}=\frac{1}{4} W=-\frac{g}{4}\left(\mathrm{e}^{-3 x}+3 \mathrm{e}^{x}+\frac{3}{g^{2}} \mathrm{e}^{-2 h-x}\right) \\
& \dot{h}=-\frac{1}{6} \partial_{h} W=-\frac{1}{g} \mathrm{e}^{-2 h-x} \\
& \dot{x}=-\frac{1}{6} \partial_{x} W=-\frac{g}{2}\left(\mathrm{e}^{-3 x}-\mathrm{e}^{x}+\frac{1}{g^{2}} \mathrm{e}^{-2 h-x}\right) .
\end{aligned}
$$

These equations can be solved explicitly by introducing a new radial variable given by $z=\frac{1}{2} g^{2} \mathrm{e}^{2 h}$. The solution is

$$
\begin{aligned}
\mathrm{e}^{-2 x} & =\frac{\mathrm{I}_{\frac{3}{4}}[z]-c \mathrm{~K}_{\frac{3}{4}}[z]}{\mathrm{I}_{-\frac{1}{4}}[z]+c \mathrm{~K}_{\frac{1}{4}}[z]} \\
\mathrm{e}^{A+\frac{3}{2} x} & =z\left(\mathrm{I}_{-\frac{1}{4}}[z]+c \mathrm{~K}_{\frac{1}{4}}[z]\right),
\end{aligned}
$$

where $\mathrm{K}_{\nu}$ and $\mathrm{I}_{\nu}$ are the modified Bessel functions and $c$ is an integration constant. A second integration constant appears in the second equation, but it can be set to unity by a coordinate transformation in the metric.

We have plotted the various orbits of $\mathrm{e}^{-2 x}$ in Figure 1 labelled by different values of $c$. We will see in the next section that these correspond to the flows from the UV to the IR at different points in the moduli space of the gauge theory. For the moment, we simply note that there are three distinct regions. For $c \geq 0$ there is a singularity in the solution at finite $z=z_{0} \geq 0$ where $\mathrm{e}^{-2 x}$ vanishes. For $-\sqrt{2} / \pi \leq c<0$ the singularity occurs at $z=z_{0}=0$, and now $\mathrm{e}^{-2 x}$ diverges. For $c<-\sqrt{2} / \pi$ there is again a singularity where $\mathrm{e}^{-2 x}$ diverges but now at a finite non-zero value of $z$.

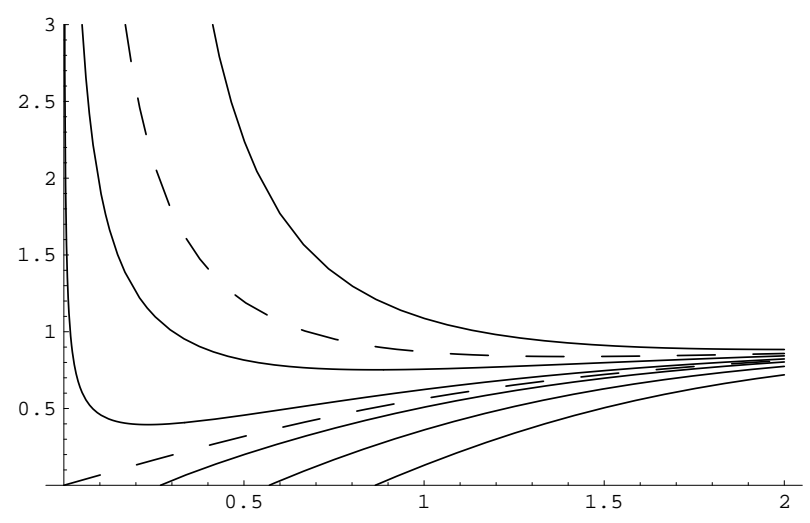

Figure 1: Plot of $\mathrm{e}^{-2 x}$ versus the radial coordinate $z$, for the three-sphere case. The lower dashed line corresponds to $c=0$, whereas the upper one corresponds to $c=$ $-\sqrt{2} / \pi$. 
We can easily obtain solutions replacing the three-sphere with the hyperbolic space $H^{3}$ or quotients thereof. The only change occurring in the Lagrangian (6) is the sign in front of the first term of the potential, induced by the negative curvature. The solution for first order equations is obtained by formally replacing $g^{2} \rightarrow-g^{2}$, and results in an overall sign change in (10). This means that the hyperbolic space case corresponds to the negative branch of the very same solutions depicted in Figure 1 and we have plotted them in Figure 2. As the dual field-theory interpretation of these solutions is not clear we will not discuss them further in the following and instead focus on the three-sphere case.

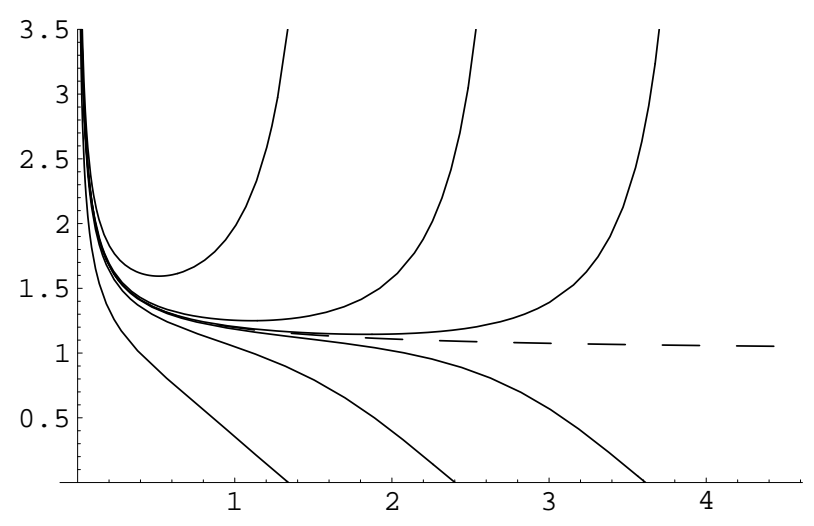

Figure 2: Plot of $\mathrm{e}^{-2 x}$ versus the radial coordinate $z$, for the hyperbolic case. The dashed line corresponds to $c \rightarrow \infty$.

\subsection{The supergravity solution in $\mathrm{D}=10$}

Using the formulae given in 28] we can uplift the seven-dimensional solution to obtain the corresponding $D=10$ supergravity solution. This can be viewed as an $\mathcal{N}=1$ solution or a type IIA or IIB solution with only NS-NS fields non-vanishing.

In the string frame our family of solutions read

$$
\mathrm{d} s^{2}=\mathrm{d} \xi^{2}+\frac{2 z}{g^{2}} \mathrm{~d} \Omega_{3}^{2}+\frac{\mathrm{e}^{2 x}}{g^{2}}\left(\mathrm{~d} z^{2}+\mathrm{d} \psi^{2}\right)+\frac{1}{g^{2} \Omega} \sin ^{2} \psi\left(E_{1}^{2}+E_{2}^{2}\right)
$$

where

$$
\begin{aligned}
& E_{1}=\mathrm{d} \theta+\cos \phi \frac{\sigma^{1}}{2}-\sin \phi \frac{\sigma^{2}}{2} \\
& E_{2}=\sin \theta\left(\mathrm{d} \phi+\frac{\sigma^{3}}{2}\right)-\cos \theta\left(\sin \phi \frac{\sigma^{1}}{2}+\cos \phi \frac{\sigma^{2}}{2}\right)
\end{aligned}
$$

and

$$
\Omega=\mathrm{e}^{2 x} \sin ^{2} \psi+\mathrm{e}^{-2 x} \cos ^{2} \psi
$$


As before, the coordinates $\left\{\xi^{i}\right\}, i=0,1,2$, parameterise the Minkowski space on the unwrapped world volume directions of the fivebrane while $\mathrm{d} \Omega_{3}^{2}$ is the metric on the three-sphere on which the fivebrane is wrapped and is expressed in terms of the $S U(2)$ left-invariant one-forms $\sigma^{a}$. The coordinates $0 \leq \psi \leq \pi, 0 \leq \theta \leq \pi, 0 \leq \phi<2 \pi$ parameterize the squashed and twisted three-sphere transverse to the fivebrane.

The dilaton is given by

$$
\mathrm{e}^{-2 \Phi+2 \Phi_{0}}=\Omega \sqrt{z}\left(\mathrm{I}_{\frac{3}{4}}[z]-c \mathrm{~K}_{\frac{3}{4}}[z]\right)^{2}
$$

and the NS three-form has the form

$$
\begin{gathered}
H=-\frac{2 \sin ^{2} \psi}{g^{2} \Omega^{2}}\left[\left(2 \cos ^{2} \psi \sinh ^{2} 2 x-\mathrm{e}^{2 x} \cosh 2 x\right) \mathrm{d} \psi\right. \\
\left.+\cos \psi \sin \psi\left(2 \sinh 2 x-\frac{1}{2 z}\right) \mathrm{d} z\right] E_{1} E_{2} \\
-\frac{\sin \psi \cos \psi \mathrm{e}^{-2 x}}{4 g^{2} \Omega}\left[-\sin \theta \sigma^{1} \sigma^{2} E^{1}+\sigma^{3} \sigma^{1}\left(\cos \theta \cos \phi E^{1}-\sin \phi E^{2}\right)\right. \\
\left.+\sigma^{2} \sigma^{3}\left(\cos \theta \sin \phi E^{1}+\cos \phi E^{2}\right)\right] \\
-\frac{1}{4 g^{2}}\left[\cos \theta \sigma^{1} \sigma^{2}+\sin \theta \cos \phi \sigma^{3} \sigma^{1}+\sin \theta \sin \phi \sigma^{2} \sigma^{3}\right] \mathrm{d} \psi
\end{gathered}
$$

where the wedge product of forms is understood. Note that the solution depends on two parameters, the expectation value of the dilaton, $\Phi_{0}$ and the integration constant $c$ appearing in $\mathrm{e}^{-2 x}$ as given in (10).

\section{Supersymmetry and $G_{2}$ structure}

We have checked that our solution preserves two of the 16 supercharges in the $S O(4)$ gauged supergravity in $D=7$ and consequently also preserves two supercharges in type I supergravity in $D=10$, and four supercharges as a type IIA or IIB solution. It is illuminating to see how this works in $D=10$ as this will allow us to elucidate an interesting notion of generalised calibration. We will concentrate on the case of type IIB, though the corresponding type I and type IIA solutions admit a similar analysis.

We first introduce a rather non-obvious orthonormal frame given by

$$
\begin{aligned}
e^{a} & =\frac{\sqrt{2 z}}{g} S^{a}, \quad a=1,2,3 & e^{7} & =\frac{1}{g \Omega^{1 / 2}}\left(\cos \psi \mathrm{d} z-\mathrm{e}^{2 x} \sin \psi \mathrm{d} \psi\right) \\
e^{4} & =\frac{1}{g \Omega^{1 / 2}}\left(\sin \psi \mathrm{e}^{2 x} \mathrm{~d} z+\cos \psi \mathrm{d} \psi\right) & e^{8} & =\mathrm{d} \xi^{1} \\
e^{5} & =\frac{1}{g \Omega^{1 / 2}} \sin \psi E_{1} & e^{9} & =\mathrm{d} \xi^{2} \\
e^{6} & =\frac{1}{g \Omega^{1 / 2}} \sin \psi E_{2} & e^{0} & =\mathrm{d} \xi^{0}
\end{aligned}
$$


where the one-forms $S^{a}$ are defined as

$$
\begin{aligned}
S^{1} & =\cos \phi \frac{\sigma^{1}}{2}-\sin \phi \frac{\sigma^{2}}{2} \\
S^{2} & =\sin \theta \frac{\sigma^{3}}{2}-\cos \theta\left(\sin \phi \frac{\sigma^{1}}{2}+\cos \phi \frac{\sigma^{2}}{2}\right) \\
S^{3} & =-\cos \theta \frac{\sigma^{3}}{2}-\sin \theta\left(\sin \phi \frac{\sigma^{1}}{2}+\cos \phi \frac{\sigma^{2}}{2}\right) .
\end{aligned}
$$

Note that $e^{4}$ and $e^{7}$ of this frame incorporate a rotation of $\mathrm{d} z$ and $\mathrm{d} \psi$. Geometrically they describe two radial directions, one in the space transverse to the brane within the Calabi-Yau threefold and one in the remaining overall transverse direction. A similar frame was used in [12, 14]. In addition we note that $S^{1}, S^{2}$ and $S^{3}$ are related to $\sigma^{1}, \sigma^{2}$ and $\sigma^{3}$ by an $S O(3)$ rotation parametrised by the coordinates $\theta, \phi$ of the transverse three-sphere. In this frame the NS three-form is given by

$$
\begin{aligned}
H= & \frac{g \mathrm{e}^{-2 x}}{2 z \Omega^{1 / 2}}\left[\cos \psi\left(e^{124}-e^{236}-e^{135}\right)-\mathrm{e}^{2 x} \sin \psi e^{127}\right] \\
& -\frac{g \mathrm{e}^{-2 x} \sin \psi}{\Omega^{3 / 2}}\left[\mathrm{e}^{6 x} \sin ^{2} \psi+\mathrm{e}^{2 x}\left(4 \cos ^{2} \psi+1\right)-3 \mathrm{e}^{-2 x} \cos ^{2} \psi-\frac{1}{z} \cos ^{2} \psi\right] e^{567} \\
& -\frac{g \mathrm{e}^{-2 x} \cos \psi}{\Omega^{3 / 2}}\left[\mathrm{e}^{4 x} \sin ^{2} \psi-3+\mathrm{e}^{-4 x} \cos ^{2} \psi-\frac{\mathrm{e}^{2 x}}{z} \sin ^{2} \psi\right] e^{456}
\end{aligned}
$$

where $e^{m n p}=e^{m} \wedge e^{n} \wedge e^{p}$.

The type IIB supersymmetry transformations are given by

$$
\begin{aligned}
\delta \lambda & =\Gamma^{\mu} \partial_{\mu} \Phi \tau_{3} \boldsymbol{\epsilon}-\frac{1}{12} H_{\mu \nu \rho} \Gamma^{\mu \nu \rho} \boldsymbol{\epsilon}=0 \\
\delta \psi_{\mu} & =\nabla_{\mu} \boldsymbol{\epsilon}-\frac{1}{8} H_{\mu \nu \rho} \Gamma^{\nu \rho} \tau_{3} \boldsymbol{\epsilon}=0,
\end{aligned}
$$

where $\boldsymbol{\epsilon}=\left(\epsilon^{-}, \epsilon^{+}\right)$is the $S O(2)$-doublet of chiral IIB supersymmetry parameters and $\tau_{3}$ is the third Pauli matrix. Note that the gravitino variation can be written in component form as

$$
\nabla^{-} \epsilon^{-}=0, \quad \nabla^{+} \epsilon^{+}=0
$$

where we introduce a generalized connection with totally antisymmetric torsion, given by

$$
\nabla_{\mu}^{ \pm}=\nabla_{\mu} \pm \frac{1}{8} H_{\mu \nu \rho} \Gamma^{\nu \rho}
$$

where $\nabla_{\mu}$ is the Levi-Civita connection. From the dilatino variation we infer the following projections on the preserved supersymmetry

$$
\begin{aligned}
& \Gamma^{1256} \boldsymbol{\epsilon}=\boldsymbol{\epsilon} \\
& \Gamma^{1346} \boldsymbol{\epsilon}=\boldsymbol{\epsilon} \\
& \Gamma^{4567} \boldsymbol{\epsilon}=\tau_{3} \boldsymbol{\epsilon} .
\end{aligned}
$$


We will not give the angular dependence of the spinors which can be determined by examining the gravitino variation. These projections preserve four independent Killing spinors, corresponding to $\mathcal{N}=2$ in $D=3$. Note that the first two projections are the same as those for Killing spinors on a Calabi-Yau threefold with tangent directions $\{1,2,3,4,5,6\}$ and the third projection for a IIB NS fivebrane with tangent directions $\{0,1,2,3,8,9\}$. In other words, the supersymmetry preserved matches that of a NS fivebrane probe wrapping a SLAG three-cycle in a Calabi-Yau threefold as expected. Note that the analogous analysis shows that as a solution of type IIA it also preserves four supercharges while as a solution of type I it preserves just two.

The projections (23) correspond to those of a $G_{2}$ holonomy manifold with tangent directions $\{1,2,3,4,5,6,7\}$. The presence of $\tau_{3}$ means that the projections for $\epsilon^{-}$and $\epsilon^{+}$differ by a sign. The two are related by simply reversing the sign of $e^{7}$ in the orthonormal frame (17). This structure gives us an alternative interpretation of the solution, which is discussed in more generality in section 7 . The seven-dimensional part of the metric can be viewed a $G_{2}$ holonomy manifold with totally antisymmetric torsion. In fact, the manifold admits two distinct covariantly constant spinors $\epsilon^{-}$and $\epsilon^{+}$with respect to two distinct connections with totally antisymmetric torsion, $\nabla^{ \pm}$, each of which has $G_{2}$ holonomy. The presence of $G_{2}$ holonomy can be characterised by the existence of a covariantly constant associative three-form $\phi$. This can be constructed from the covariantly constant spinor $\epsilon$ by

$$
\phi_{\alpha \beta \gamma}=\epsilon^{\mathrm{T}} \gamma_{\alpha \beta \gamma} \epsilon
$$

where $\gamma_{\alpha}$ are gamma matrices on the $G_{2}$ holonomy manifold, and we normalize $\epsilon$ by $\epsilon^{\mathrm{T}} \epsilon=1$. Thus, since we have two different spinors $\epsilon^{ \pm}$, we can construct two different associative three-forms. Given the projections (23), these can be immediately written down in the frame (17), giving

$$
\begin{aligned}
& \phi^{-}=e^{123}+e^{145}-e^{167}+e^{246}+e^{257}+e^{347}-e^{356} \\
& \phi^{+}=-e^{123}-e^{145}-e^{167}-e^{246}+e^{257}+e^{347}+e^{356} .
\end{aligned}
$$

Since $\nabla^{-} \epsilon^{-}=0$ and $\nabla^{+} \epsilon^{+}=0$, then, by construction, the associated three-forms are covariant constant but with respect to different connections

$$
\begin{aligned}
& \nabla^{-} \phi^{-}=0 \\
& \nabla^{+} \phi^{+}=0 .
\end{aligned}
$$

We should also be able to view the associative three-forms $\phi^{ \pm}$as a generalized calibration. Again this will be discussed in more detail in section 07. For the moment we simply note that using the explicit expressions (19) and (25), one can derive an expression for the dual NS six-form potential $\tilde{B}$ defined by $\mathrm{d} \tilde{B} \equiv \tilde{H}=* \mathrm{e}^{-2 \Phi} H$. We find that

$$
\tilde{B}= \pm \mathrm{Vol}_{3} \wedge \mathrm{e}^{-2 \Phi} \phi^{ \pm}
$$

where $\mathrm{Vol}_{3}$ is the volume form of the unwrapped part of the fivebrane world-volume. This holds for either choice of $\phi^{ \pm}$, the two expressions differing by a gauge transformation. This expression for the dual potential will be particularly useful when we 
come to consider a probe calculation in section 6. It is equivalent to

$$
\mathrm{d}\left(\mathrm{Vol}_{3} \wedge \mathrm{e}^{-2 \Phi} \phi^{ \pm}\right)= \pm \tilde{H}
$$

which, as we will show, can be viewed as the generalized calibration condition on a $G_{2}$ holonomy manifold with torsion and a non-trivial dilation field.

\section{$5 \quad$ UV and IR limits}

Let us now discuss the asymptotic UV and IR behaviour of our solutions. The UV limit is obtained when $z \rightarrow \infty$. The metric and dilaton then take the form

$$
\begin{aligned}
\mathrm{d} s^{2} & \approx \mathrm{d} \xi^{2}+\frac{2 z}{g^{2}} \mathrm{~d} \Omega_{3}^{2}+\frac{1}{g^{2}}\left[\mathrm{~d} z^{2}+\mathrm{d} \psi^{2}+\sin ^{2} \psi\left(E_{1}^{2}+E_{2}^{2}\right)\right] \\
\mathrm{e}^{-2 \Phi+2 \Phi_{0}} & \approx \frac{1}{2 \pi \sqrt{z}} \mathrm{e}^{2 z}
\end{aligned}
$$

This has the same form as the near horizon limit of the flat NS-fivebrane solution (1) but with world volume $\mathbb{R}^{1,2} \times S^{3}$ instead of $\mathbb{R}^{1,5}$ and the appropriate twisting. Note that the dilaton is asymptotically linear up to a logarithmic correction.

The one parameter family of solutions, specified by $c$, are all singular in the IR, that is for $z \approx z_{0}$, where $z_{0}$ is defined where $\mathrm{e}^{-2 x}$ goes to zero or diverges, depending on the value of $c$ (see Figure 1). This can be seen by analysing the behaviour of the dilaton, which is plotted in Figure 3 (for $\psi=0$ ). For the solutions with $c<0$, it blows up at $z_{0}$ and generic values of $\psi$ while for the solutions with $c \geq 0$ it vanishes at $z_{0}$ for generic $\psi$. Since the $g_{t t}$ component of the Einstein frame metric is given by $\mathrm{e}^{-\Phi / 2}$, these singularities are of the "good" type for $c \geq 0$ and of the "bad" type for $c<0$, using the criteria of [2]. We thus expect that only the solutions with $c \geq 0$ are associated with $\mathcal{N}=2 D=3$ Yang-Mills theory phenomena.

We now analyse the limiting form of the metric near the good singularities. First notice that the function $\mathrm{I}_{-\frac{1}{4}}+c \mathrm{~K}_{\frac{1}{4}}$ is positive definite for $c \geq 0$, and hence a zero of $\mathrm{e}^{-2 x}$ occurs when

$$
\mathrm{I}_{\frac{3}{4}}\left[z_{0}\right]-c \mathrm{~K}_{\frac{3}{4}}\left[z_{0}\right] \equiv 0
$$

Next, using (30), and recursion relations satisfied by the modified Bessel functions 29, we find, near $z_{0}$

$$
\mathrm{e}^{-2 x} \approx \begin{cases}\frac{2}{3} z & \text { for } \quad c=0 \\ z-z_{0} & \text { for } \quad c>0\end{cases}
$$

We also need to know the following asymptotic expansion near $z_{0}$

$$
\mathrm{I}_{\frac{3}{4}}[z]-c \mathrm{~K}_{\frac{3}{4}}[z] \approx \begin{cases}\frac{2^{3 / 4}}{3 \Gamma\left(\frac{3}{4}\right)} z^{3 / 4} & \text { for } c=0 \\ \gamma\left(z-z_{0}\right) & \text { for } \quad c>0\end{cases}
$$




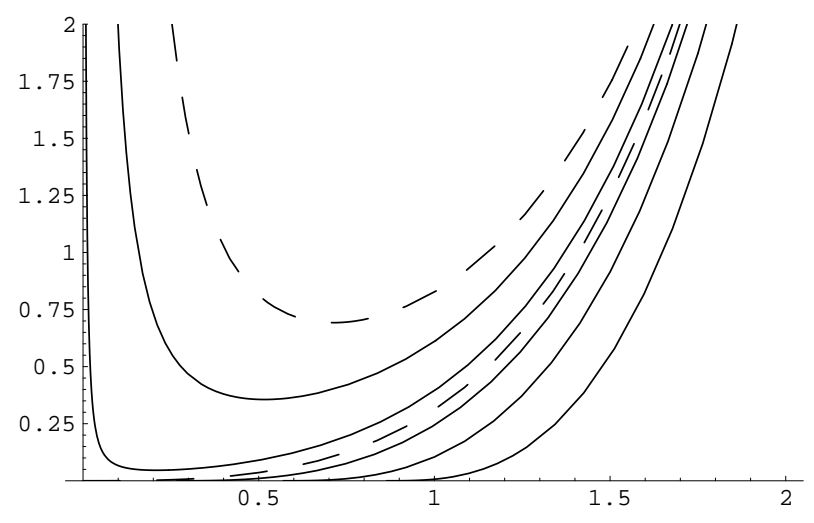

Figure 3: Plot of $\mathrm{e}^{-2 \Phi}$ versus the radial coordinate $z$. The lower dashed line corresponds to $c=0$, whereas the upper one corresponds to $c=-\sqrt{2} / \pi$.

where we have defined the positive constant $\gamma=\mathrm{I}_{-\frac{1}{4}}\left[z_{0}\right]-c \mathrm{~K}_{\frac{1}{4}}\left[z_{0}\right]$, depending on $c$. The IR behaviour of the solutions can now be written down, and for simplicity we do so just for the $c>0$ solutions. If we define $y=z-z_{0}$, we find (for $\psi \neq 0, \pi$ ) the IR limit

$$
\begin{aligned}
\mathrm{d} s^{2} & \approx \mathrm{d} \xi^{2}+\frac{2 z_{0}}{g^{2}} \mathrm{~d} \Omega_{3}^{2}+\frac{1}{g^{2} y}\left[\mathrm{~d} y^{2}+y^{2}\left(E_{1}^{2}+E_{2}^{2}\right)+\mathrm{d} \psi^{2}\right] \\
\mathrm{e}^{-2 \Phi+2 \Phi_{0}} & \approx \gamma^{2} \sqrt{z_{0}} y \sin ^{2} \psi
\end{aligned}
$$

The form of the metric indicates that the singularity corresponds to a linear distribution of fivebranes. This is parameterized by the angle $\psi$ which varies in $0 \leq \psi \leq \pi$ and it has therefore the topology of a segment. The origin of the $\sin ^{2} \psi$ factor in the expression for the dilaton is not clear, as one expects the same harmonic function, in this case $1 / y$ appearing in the metric and $\mathrm{e}^{2 \Phi}$. Presumably it means one needs to take the limit more carefully. However, we can give some more evidence for the picture advocated, by studying the limit near $\psi=0, \pi$ where the above expansion is certainly not valid. In this case we find it useful to introduce a change of variables similar to one performed in [14]. Near $\psi=0$, for instance, let

$$
\begin{aligned}
& y=\sqrt{\rho} \sin \frac{\alpha}{2} \\
& \psi=\sqrt{\rho} \cos \frac{\alpha}{2}
\end{aligned}
$$

from which the expansions take the form

$$
\begin{aligned}
\mathrm{d} s^{2} & \approx \mathrm{d} \xi^{2}+\frac{2 z_{0}}{g^{2}} \mathrm{~d} \Omega_{3}^{2}+\frac{1}{4 g^{2} \rho^{3 / 2} \sin (\alpha / 2)}\left(\mathrm{d} \rho^{2}+\rho^{2}\left[\mathrm{~d} \alpha^{2}+\sin ^{2} \alpha\left(E_{1}^{2}+E_{2}^{2}\right)\right]\right) \\
\mathrm{e}^{-2 \Phi+2 \Phi_{0}} & \approx \gamma^{2} \sqrt{z_{0}} \rho^{3 / 2} \sin (\alpha / 2)
\end{aligned}
$$


and note that $1 / \rho^{3 / 2} \sin (\alpha / 2)$ is harmonic in $\mathbb{R}^{4}$. Keeping $\rho$ small, but finite, the above expressions show that the singularity occurs for $\alpha=0$, which is the segment near its end point.

\section{A probe computation}

We would like to argue that the family of solutions corresponding to good singularities $(c \geq 0)$ should describe a slice of the Coulomb branch of $D=3 \mathcal{N}=2$ pure Yang-Mills theory. It is known that the perturbative Coulomb branch of this theory is unstable due to the generation of a superpotential [18, 19, 20]. However, this superpotential is induced by instanton effects, and it has been observed in previous cases [14, 30] that such effects are not captured by the supergravity approximation. Thus, it is not unexpected that we can find a supergravity solution dual to the perturbative Coulomb branch of the gauge theory.

We shall provide evidence for our interpretation by probing our solutions with a fivebrane. The $U(1)$ dynamics of the probe fivebrane corresponds in the field theory language to Higgsing the gauge group $S U(N) \rightarrow S U(N-1) \times U(1)$ and analysing the dynamics of the $U(1)$ factor. To properly treat the IR dynamics of the gauge theory on the NS fivebranes one should switch to an S-dual description in terms of D-fivebranes [31]. The D5 brane solution is obtained via the following transformation rules

$$
\begin{aligned}
\Phi_{\mathrm{D} 5} & =-\Phi_{\mathrm{NS} 5} \\
\mathrm{~d} s^{2}(\mathrm{D} 5) & =\mathrm{e}^{-\Phi_{\mathrm{NS} 5}} \mathrm{~d} s^{2}(\mathrm{NS} 5) \\
C_{(2)} & =-B
\end{aligned}
$$

where the quantities on the left hand side refer to the S-dual dilaton, metric, and RR potential. Similarly, the corresponding six-form potential dual to $C_{(2)}$ is given by $C_{(6)}=-\tilde{B}$.

The effective action of a probe D5 brane in the string frame reads

$$
\begin{aligned}
S=-\mu_{5} & \int \mathrm{d}^{6} y \mathrm{e}^{-\Phi_{\mathrm{D} 5}} \sqrt{-\operatorname{det}\left[G_{a b}+B_{a b}+2 \pi \alpha^{\prime} F_{a b}\right]} \\
& +\mu_{5} \int\left[\exp \left(2 \pi \alpha^{\prime} F+B\right) \wedge \oplus_{n} C_{(n)}\right]
\end{aligned}
$$

where $\mu_{5}=(2 \pi)^{-5} \alpha^{\prime-3}, F_{a b}$ is a world-volume Abelian gauge field, $B$ is the NS two-form, $C_{(n)}$ are the RR $n$-forms and it is understood that ten-dimensional fields are pulled back to the six-dimensional world volume. The S-dual D5-brane solution has $B=0$, and non-vanishing $C_{(2)}$ and $C_{(6)}$. However, one immediately sees that the pullback of the three-form has no components on the three-sphere on which the brane is wrapped, which means that there is no Chern-Simons term in the corresponding gauge theory.

We choose the world-volume to have topology $\mathbb{R}^{1,2} \times S^{3}$ and fix reparametrisation invariance by identifying the world-volume coordinates with $\left\{\xi^{i}, \tilde{\psi}, \tilde{\theta}, \tilde{\phi}\right\}$, where the 
tilded angles are coordinates on the three-sphere on which the brane is wrapped. The four scalar fields $z, \psi, \theta$, and $\phi$ are then functions of these coordinates. The dynamics of the fivebrane relevant for the three-dimensional gauge theory is obtained by letting the fields depend only on the non-compact coordinates $\left\{\xi^{i}\right\}$. In order to find which moduli do not break supersymmetry, let us first consider the static part of the action, setting $\{z, \psi, \theta, \phi\}$ to constants and $F=0$. The contribution from the WZ part is easily obtained from (27) and reads

$$
S_{\mathrm{WZ}}=2 \pi^{2} \mu_{5}\left(\frac{2 z}{g^{2}}\right)^{3 / 2} \int \mathrm{d}^{3} \xi \mathrm{e}^{-2 \Phi} .
$$

The static contribution from the DBI determinant is in general rather complicated due to contributions from the twisted part of the metric (contained in $E_{1}$ and $E_{2}$ ), however it is not difficult to verify that it cancels against the WZ part if we restrict to $\psi=0, \pi$ or to $z=z_{0}$, in which case the two terms vanish separately due to the vanishing of the dilaton at the singularity. We therefore identify two distinct loci for supersymmetric motion of the wrapped brane (cf. [14]).

We next consider non-zero velocities for the fields in order to find the moduli space metric on the two loci. First, let us treat the gauge field. We are interested in turning on a gauge field in the unwrapped directions of the brane. After integrating over the three-sphere that the brane wraps, we can dualise the $D=3 U(1)$ gauge-field (see e.g. 34) to get a compact scalar field. The $\phi$ and $\theta$ kinetic terms vanish upon restricting to the supersymmetric loci, leaving the following non trivial contributions in each of the two cases

$$
S_{\mathrm{I}}=-\int \mathrm{d}^{3} \xi\left[c_{1} z^{2}\left(\mathrm{I}_{\frac{3}{4}}[z]-c \mathrm{~K}_{\frac{3}{4}}[z]\right)^{2}(\partial z)^{2}+c_{2} z^{-3 / 2}(\partial \sigma)^{2}\right] \quad(\psi=0, \pi)
$$

valid for $c \geq 0$, and

$$
S_{\mathrm{II}}=-\int \mathrm{d}^{3} \xi\left[c_{1} \gamma^{2} z_{0}^{2} \sin ^{2} \psi(\partial \psi)^{2}+c_{2} z_{0}^{-3 / 2}(\partial \sigma)^{2}\right] \quad\left(z=z_{0}\right)
$$

valid for $c>0$. The constants $c_{1,2}$ are defined as follows

$$
c_{1}=\frac{\mathrm{e}^{-2 \Phi_{0}}}{\pi^{3} 2^{7 / 2} g^{5}}, \quad c_{2}=2^{3 / 2} \pi^{3} g^{3} .
$$

Let us describe the resulting moduli space, focusing on the $c>0$ case. Locus II has the topology of a cylinder and it covers the space-time singularity. The moduli space metric on the cylinder is flat as can be seen by employing the change of coordinates $\zeta=\cos \psi$, giving

$$
\mathrm{d} s_{\mathcal{M}_{I I}}^{2}=c_{1} z_{0}^{2} \gamma^{2} \mathrm{~d} \zeta^{2}+c_{2} z_{0}^{-3 / 2} \mathrm{~d} \sigma^{2}
$$

On the other hand, locus I consists of two disconnected parts $(\psi=0$ and $\psi=\pi)$, each of which is a cylinder whose radius is finite near the singularity and goes to zero 
as $z \rightarrow \infty$. By performing the change of coordinates $\eta=1 / 2\left(z-z_{0}\right)^{2}$ we can use the expansion (32) to obtain the following expression for the metric on locus I near the singularity

$$
\mathrm{d} s_{\mathcal{M}_{I}}^{2} \simeq c_{1} z_{0}^{2} \gamma^{2} \mathrm{~d} \eta^{2}+c_{2} z_{0}^{-3 / 2} \mathrm{~d} \sigma^{2} \quad \text { for } \quad z \rightarrow z_{0}
$$

We notice that, in this limit, the radius of each of the two cylinders of Locus I is identical to that of Locus II. This suggests, as in [14], that we identify the three pieces along the boundaries, resulting in a single infinite "cylinder" both ends of which are shrinking to zero as $z \rightarrow \infty$. Note that the moduli space is smooth even where the space-time is singular.

\section{Geometry of wrapped fivebranes and generalised calibrations}

In this section we will further elucidate the types of geometry that arise when IIB fivebranes wrap supersymmetric cycles in special holonomy manifolds $X$. The results extend simply to type IIA by T-duality and to type I in an obvious way. To be explicit one starts by considering a probe brane in a geometry $\mathbb{R}^{1, p} \times\left(X \times \mathbb{R}^{q}\right)$. Here the first factor represents the unwrapped brane directions. The brane is taken to be wrapped on some cycle $\Sigma$ in $X$. The factor $\mathbb{R}^{q}$ simply represents the remaining "overall transverse" directions to the brane, making the total space ten-dimensional.

In all cases, in the string frame, the back-reaction of the brane on the geometry preseves the flat $\mathbb{R}^{1, p}$ factor but promotes $X \times \mathbb{R}^{q}$ to a non-trivial Riemannian manifold $Y$ with non-vanishing dilaton and NS three-form. Moreover, in general, $Y$ has two connections with totally anti-symmetric torsion given by $\nabla^{ \pm}=\nabla \pm \frac{1}{2} H$, as in (22). We will see that in some cases both of these have special holonomy while in others just one of them has special holonomy. The supersymmetry transformations in type IIB are parametrised by a doublet of Majorana-Weyl $S O(1,9)$ spinors $\boldsymbol{\epsilon}=\left(\epsilon^{-}, \epsilon^{+}\right)$ with the same chirality. In each case the structure on $Y$ can be inferred from the projections on the Killing spinors coming from, first, the special holonomy on $X$ and, second, the additional projection implied by the presence of a brane. The latter is different for $\epsilon^{-}$and $\epsilon^{+}$. The cases where only one of $\nabla^{-}$or $\nabla^{+}$have special holonomy on $Y$ occurs when the two different projections on $\epsilon^{-}$and $\epsilon^{+}$implied by the brane combined with the geometrical projections implied by $X$ only preserve $\epsilon^{-}$ or $\epsilon^{+}$spinors. Cases where both $\nabla^{+}$and $\nabla^{-}$have special holonomy on $Y$ occur when the combined projections preserve both $\epsilon^{-}$and $\epsilon^{+}$spinors. In particular, if there is an overall transverse direction when a fivebrane wraps the supersymmetric cycle, i.e. $q$ is non-zero, then there are always two structures.

Let us first dicuss the seven-dimensional case where $Y$ has $G_{2}$ holonomy and then turn to the other cases. In this paper we have presented a solution describing a fivebrane wrapping a SLAG three-cycle in a Calabi-Yau threefold $X$. This is a case where there is one overall transverse direction when a probe fivebrane wraps the cycle. In the full solution, including the back-reaction, the space no longer has a 
product structure, but the decomposition into $X$ and an overall transverse direction is nonetheless still encoded in the supersymmetry projections. In particular, the supersymmetry projections for the parallel spinors of a Calabi-Yau threefold can be written, as in eqn. (23) with some relabelling, as

$$
\begin{aligned}
& \Gamma^{1234} \boldsymbol{\epsilon}=\boldsymbol{\epsilon} \\
& \Gamma^{3456} \boldsymbol{\epsilon}=\boldsymbol{\epsilon} .
\end{aligned}
$$

If we decompose each $S O(1,9)$ Majorana-Weyl spinor $\epsilon^{ \pm}$into $S O(1,3) \times S U(3) \subset$ $S O(3,1) \times \operatorname{Spin}(6)$ representations, these projections imply that the preserved spinors are $S U(3)$ singlets. As usual this gives eight supercharges or $\mathcal{N}=2$ in $D=4$. The presence of a probe fivebrane implies the extra projection

$$
\Gamma^{1357} \boldsymbol{\epsilon}=\tau_{3} \boldsymbol{\epsilon} .
$$

As we have seen these projections are satisfied by the Killing spinors of our particular solution. However, the analysis is quite general. Any supergravity solution representing a fivebrane wrapping a SLAG cycle will incorporate the same projections. Many features of the particular solution then go through. First decompose each $S O(1,9)$ Majorana-Weyl spinor into $S O(1,2) \times \operatorname{Spin}(7)$ via $\mathbf{1 6}_{+} \rightarrow(\mathbf{2}, \mathbf{8})$, and with a slight abuse of notation, let $\boldsymbol{\epsilon}$ also denote the doublet $\left(\epsilon^{-}, \epsilon^{+}\right)$of seven-dimensional spinors. The above projections imply that for each $\epsilon^{ \pm}$there is a $G_{2}$ subgroup of $\operatorname{Spin}(7)$ with $\mathbf{8} \rightarrow \mathbf{7}+\mathbf{1}$ with $\epsilon^{ \pm}$transforming as the singlet. Note that the presence of $\tau_{3}$ implies that the projections are different for each $\epsilon^{ \pm}$(related by simply reversing the sign of the overall transverse tangent direction $e^{7}$ ) and hence the $G_{2}$ subgroups are different (related by an outer automorphism). Since the Killing spinors satisfy $\nabla^{-} \epsilon^{-}=0$ and $\nabla^{+} \epsilon^{+}=0$ (see (20)), the holonomy of the two connections $\nabla^{ \pm}$are each separately in $G_{2}$. This implies that one can construct two different associative three-forms on $Y$, given by

$$
\phi_{\alpha \beta \gamma}^{ \pm}=\epsilon^{ \pm \mathrm{T}} \gamma_{\alpha \beta \gamma} \epsilon^{ \pm}
$$

where $\gamma^{\alpha}$ are seven-dimensional gamma matrices, and we normalise $\epsilon^{ \pm}$by $\epsilon^{ \pm \mathrm{T}} \epsilon^{ \pm}=1$. By construction each associative threeform is covariantly constant but with respect to different connections with torsion

$$
\nabla^{-} \phi^{-}=0, \quad \nabla^{+} \phi^{+}=0 .
$$

In summary, we see that the supersymmetry projections on the spinors on the CalabiYau threefold $X$ together with the projection for the probe fivebrane imply that $Y$ admits two different connections with $G_{2}$ holonomy and we have four conserved supercharges, corresponding to $\mathcal{N}=2$ in $D=3$ on the unwrapped part of the fivebrane world-volume.

By contrast let us consider probe fivebranes wrapping associative three-cycles in a seven-dimensional manifold $X$ of $G_{2}$ holonomy. Explicit solutions for this case were 
given in 15, 10, 11]. In this case $q=0$ and there is no overall transverse direction. The $G_{2}$ holonomy of $X$ implies the supersymmetry projections

$$
\begin{aligned}
& \Gamma^{1256} \boldsymbol{\epsilon}=-\boldsymbol{\epsilon} \\
& \Gamma^{1357} \boldsymbol{\epsilon}=\boldsymbol{\epsilon} \\
& \Gamma^{1467} \boldsymbol{\epsilon}=\boldsymbol{\epsilon} .
\end{aligned}
$$

As above, decomposing $S O(1,9)$ spinors into $S O(2,1) \times \operatorname{Spin}(7)$, these projections pick out the singlet representation of $G_{2} \subset \operatorname{Spin}(7)$, and give two conserved supercharges for $\epsilon^{-}$and for $\epsilon^{+}$, corresponding to $\mathcal{N}=2$ in $D=3$. As before, the presence of a probe fivebrane implies the extra projection

$$
\Gamma^{1357} \boldsymbol{\epsilon}=\tau_{3} \boldsymbol{\epsilon} .
$$

Unlike the previous case, this is only compatible with the $G_{2}$ projections (50) for $\epsilon^{-}$and not for $\epsilon^{+}$. Thus only two supercharges survive corresponding to $\mathcal{N}=1$ in $D=3$ on the unwrapped part of the fivebrane. As a result, solutions that include the back-reaction on the geometry give a new seven manifold $Y$ with torsion, and only one Killing spinor $\nabla^{-} \epsilon^{-}=0$. That is, in contrast to the previous case, there is now only one connection on $Y$ with $G_{2}$ holonomy. Consequently there is only one associative three-form $\phi^{-}$satisfying, by construction, $\nabla^{-} \phi^{-}=0$. Indeed the explicit solutions constructed in [5, 10, 11] are of this type and it is worth emphasizing that the solutions in 10, 11] are non-singular examples of this kind of geometry.

Before considering the cases of other wrapped NS fivebranes, let us return to the issue of generalised calibrations mentioned at the end of section 4 . Let us first recall the notion of calibration. On a manifold of $G_{2}$ holonomy the associative three-form $\phi$ is a calibration in that it satisfies the following conditions. First, if pulled back onto any tangent three-plane it is less than or equal to the induced volume-form evaluated on the three-plane. Secondly, it is closed $\mathrm{d} \phi=0$. As a result a calibrated three-manifold whose volume-form is equal to the pull-back of the associative threeform is minimal and, essentially because the associative three-form has a spinorial construction, is supersymmetric.

If the background also includes a form-field flux, the notion of calibration must be generalised 32, 33] in order for it to characterise supersymmetric cycles. In particular, $\phi$ is no longer closed, but rather $\mathrm{d} \phi$ is related to the flux. Recall that, in section 4 , we showed that in our solution $\phi$ was related to the dual NS potential $\tilde{B}$ together with the dilaton. The result (28), can be rewritten as

$$
H=\mp \mathrm{e}^{2 \Phi} *_{7} \mathrm{~d}\left(\mathrm{e}^{-2 \Phi} \phi^{ \pm}\right)
$$

where $*_{7}$ is the Hodge dual operator on $Y$ and this is the correct generalisation of the calibration condition $\mathrm{d} \phi=0$ in the presence of both NS flux and a non-trivial dilaton. To see this, recall that the action for a NS fivebrane with zero world-volume gauge field strength and no background RR fields is given by

$$
S=-\mu_{5} \int \mathrm{d}^{6} y \mathrm{e}^{-2 \Phi} \sqrt{-G}-\mu_{5} \int \tilde{B},
$$


where $G$ is the induced world-volume metric. Let us further consider the NS fivebrane probe to be static in a spacetime of the form $\mathbb{R}^{1,2} \times Y$. One can then repeat the same steps as in [32, 33] to conclude that if the fivebrane has world-volume with volume form given by the pull-back of $\mathrm{Vol}_{3} \wedge \mathrm{e}^{-2 \Phi} \phi$ then, given the condition (52), it minimises the fivebrane static energy and, hence, $\phi$ is a generalized calibration and the probe configuration is supersymmetric.

To see the connection with supersymmetry, we now show that this condition can be derived in general from the supersymmetry conditions. Reducing to seven dimensions, the dilatino variation can be rewritten as

$$
\delta \lambda=\partial_{\alpha} \Phi \gamma^{\alpha} \tau_{3} \boldsymbol{\epsilon}-\frac{1}{12} H_{\alpha \beta \gamma} \gamma^{\alpha \beta \gamma} \boldsymbol{\epsilon}=0
$$

Given the symmetry properties of $\gamma^{\alpha}$, this implies that

$$
\begin{aligned}
& \partial_{\alpha} \Phi \epsilon^{-\mathrm{T}}\left[A, \gamma^{\alpha}\right]_{\mp} \epsilon^{-}-\frac{1}{12} H_{\alpha \beta \gamma} \epsilon^{-\mathrm{T}}\left[A, \gamma^{\alpha \beta \gamma}\right]_{ \pm} \epsilon^{-}=0 \\
& \partial_{\alpha} \Phi \epsilon^{+^{\mathrm{T}}}\left[A, \gamma^{\alpha}\right]_{\mp} \epsilon^{+}+\frac{1}{12} H_{\alpha \beta \gamma} \epsilon^{+^{\mathrm{T}}}\left[A, \gamma^{\alpha \beta \gamma}\right]_{ \pm} \epsilon^{+}=0
\end{aligned}
$$

where $A$ is an operator built out of gamma matrices and $[\cdot, \cdot]_{ \pm}$refer to the anticommutator and commutator. Consider the case where $A=\gamma_{\alpha_{1} \alpha_{2} \alpha_{3} \alpha_{4}}$ and choose the upper sign. Using the fact that in general either $\nabla^{-} \epsilon^{-}=0$ and $\nabla^{+} \epsilon^{+}=0$ or only one vanishes, and with the orientation on $Y$ given by $\epsilon^{\alpha_{1} \ldots \alpha_{7}}=\epsilon^{ \pm \mathrm{T}} \gamma^{\alpha_{1} \ldots \alpha_{7}} \epsilon^{ \pm}$, it is then easy to show that (55) implies the generalized calibration condition (52) for one or both of the associated three-forms. In particular, when both $\phi^{ \pm}$exist, each separately are generalized calibrations, while when there is just a single $G_{2}$ structure, we have a unique generalized calibration.

Thus far our discussion has focussed on the associative three-forms $\phi^{ \pm}$on $Y$. However, one can always also construct the corresponding co-associative four-forms ${ }_{*_{7}} \phi_{\alpha_{1} \ldots \alpha_{4}}^{ \pm}=\epsilon^{ \pm} \gamma_{\alpha_{1} \ldots \alpha_{4}} \epsilon^{ \pm}$which are just the Hodge duals of $\phi^{ \pm}$. It is natural to ask what generlised calibration condition the co-associative form satisfies when the torsion and dilaton are non-vanishing. It is easy to show, taking $A=\gamma_{\alpha_{1} \ldots \alpha_{5}}$ and the lower signs in $(55)$, that

$$
\mathrm{d}\left(\mathrm{e}^{-2 \Phi} *_{7} \phi^{ \pm}\right)=0
$$

Note that, unlike the case of the associative form, the generalisation does not involve the three-form $H$ and only involves the dilaton factor, which is necessary because of the corresponding factor in the static energy as in (53). The general theory of a single $G_{2}$ connection with totally anti-symmetric torsion has been recently developed in [35. A general expression for $d *_{7} \phi$ is given in [35] that is consistent with (56). In addition our expression for the torsion (52) is consistent with the revised results of [35.

Let us discuss more briefly the geometries arising for other cases of wrapped NS fivebranes. First consider six-dimensional manifolds $Y$ with connections with torsion of $S U(3)$ holonomy. The first way in which these geometries arise is when fivebranes 
wrap two-cycles in a Calabi-Yau two-fold $X$. In this case $q=2$ so there are two overall transverse directions and when the back-reaction of the fivebrane on the geometry is included the dimension of the non-trivial geometry jumps from four to six. The projections on the spinors for $X$ imply

$$
\Gamma^{1234} \boldsymbol{\epsilon}=\boldsymbol{\epsilon}
$$

so that the preserved supersymmetries are singlets under the $S U(2)$ holonomy on $X$. The projection due to the brane gives

$$
\Gamma^{3456} \boldsymbol{\epsilon}=\tau_{3} \boldsymbol{\epsilon} .
$$

Comparing with (46) we see that these do indeed correspond to the projections on parallel spinors of a manifold with $S U(3)$ holonomy, though clearly with different $S U(3)$ structures for $\epsilon^{+}$and $\epsilon^{-}$, related by exchanging $e^{5}$ and $e^{6}$. Thus we conclude that on $Y$ the two connections $\nabla^{ \pm}$each have $S U(3)$ holonomy and that we can construct two commuting complex structures $J^{ \pm}$with $\nabla^{-} J^{-}=0$ and $\nabla^{+} J^{+}=0$. (Note that it can be shown [36], in general, that for all the cases of $S U(N)$ holonomy with torsion that we discuss, the objects referred to as complex structures constructed from the spinors do indeed satisfy the conditions for $Y$ to be a complex manifold.) Since both $\epsilon^{-}$and $\epsilon^{+}$are each separately conserved, the theory preserves $\mathcal{N}=2$ supersymmetry in four dimensions. This kind of geometry was first noticed in a sigma model context in [37. In addition we can repeat the generalised calibration argument, taking $A=\gamma_{\alpha_{1} \alpha_{2} \alpha_{3}}$ in the six-dimensional expression analogous to (55). We find

$$
H=\mp \mathrm{e}^{2 \Phi} *_{6} \mathrm{~d}\left(\mathrm{e}^{-2 \Phi} J^{ \pm}\right)
$$

In fact, both of these structures were found in [14] for the particular singular solutions presented in 114, 15].

Six-dimensional geometries also arise when fivebranes wrap two-cycles in CalabiYau threefolds, in which case $q=0$ and there are no overall transverse directions. In this case, the spinor projections from the geometry of $X$ were given in (46) above. However, now the brane projection is given by (58). Clearly this is only consistent with the $X$ projections for $\epsilon^{-}$, in which case the projections continue to imply $S U(3)$ holonomy. Thus we conclude that the geometry of $Y$ which includes the back-reaction only has a single connection $\nabla^{-}$with $S U(3)$ holonomy, and thus a single complex structure with $\nabla^{-} J^{-}=0$. The preserved supersymmetry is now consequently $\mathcal{N}=1$ in $D=4$. The expression for the generalised calibration condition (59) is again satisfied for $J^{-}$. Solutions of this type were constructed in [3] and their geometry was discussed in 23. Finally, it is worth noting that a different, but consistent, expression for the torsion was found in [37, 36, 38] (in the case of a single complex structure). The virtue of (59) is that this form generalises to cases of $G_{2}$ or $\operatorname{Spin}(7)$ holonomy. It is also worth noting that, as shown in [36, 38], these geometries have holomorphic threeforms of the form $\mathrm{e}^{-2 \Phi} \omega^{ \pm}$where $\omega^{ \pm}$are constructed from the Killing spinors. These are the generalised calibrations corresponding to the special Lagrangian calibrations. 
These forms are closed, so just as for the co-associative forms in the $G_{2}$ case above, the generalisation only involves the dilaton.

Let us now turn to manifolds $Y$ with $S U(4)$ holonomy. These can be obtained in four ways: from fivebranes wrapping (i) Kähler four-cycles in Calabi-Yau threefolds, (ii) a product of two Kähler two-cycles in a product of two Calabi-Yau two-folds, (iii) Kähler four-cycles in Calabi-Yau four-folds or (iv) complex Lagrangian four-cycles in eight dimensional hyper-Kähler manifolds. The first and the third case are completely analogous to the cases with $S U(3)$ holonomy just described and it will be convenient to discuss the fourth case later. Let us begin with the first case of four-cycles in threefolds. In this case there are two overall transverse directions and the projections on the parallel spinors of $X$ are given by (46) while the projection due to the brane gives

$$
\Gamma^{5678} \boldsymbol{\epsilon}=\tau_{3} \boldsymbol{\epsilon}
$$

These are precisely the projections for $S U(4)$ holonomy on $Y$ though with different structures for the two spinors $\epsilon^{-}$and $\epsilon^{+}$. That is we have two different complex structures $J^{ \pm}$satisfying $\nabla^{-} J^{-}=0$ and $\nabla^{+} J^{+}=0$. Again, the two complex structures are related by exchanging the two overall transverse directions $e^{7}$ and $e^{8}$. Note that this implies that the eight-dimensional chirality of $\epsilon^{-}$and $\epsilon^{+}$are different. As a result the theory preserves $(2,2)$ supersymmetry in the $D=2$ unwrapped world-volume dimensions. Again an expression for the generalised calibration can be derived using $A=\gamma_{\alpha_{1} \alpha_{2} \alpha_{3} \alpha_{4} \alpha_{5}}$ in the eight-dimensional analog of (55) and reads

$$
H=\frac{1}{2} \mathrm{e}^{2 \Phi} *_{8} \mathrm{~d}\left(\mathrm{e}^{-2 \Phi} J^{ \pm} \wedge J^{ \pm}\right)
$$

Note that the difference in chirality of $\epsilon^{+}$and $\epsilon^{-}$implies that $\epsilon^{\alpha_{1} \ldots \alpha_{8}}=\epsilon^{-} \gamma^{\alpha_{1} \ldots \alpha_{8}} \epsilon^{-}=$ $-\epsilon^{+} \gamma^{\alpha_{1} \ldots \alpha_{8}} \epsilon^{+}$so there is no overall sign factor in (61). The second case involves fivebranes wrapping a product of two Kähler two-cycles in a product $X$ of two CalabiYau two-folds. The projections for $X$ are

$$
\begin{aligned}
& \Gamma^{1234} \boldsymbol{\epsilon}=\boldsymbol{\epsilon} \\
& \Gamma^{5678} \boldsymbol{\epsilon}=\boldsymbol{\epsilon},
\end{aligned}
$$

while the presence of the brane wrapping a four-cycle tangent to the 1256 directions implies

$$
\Gamma^{3478} \boldsymbol{\epsilon}=\tau_{3} \boldsymbol{\epsilon} .
$$

These imply we again have two $S U(4)$ structures. Note that in contrast to the previous case, the preserved spinors have the same $S O(8)$ chirality, i.e. $\Gamma^{12345678} \boldsymbol{\epsilon}=\boldsymbol{\epsilon}$, and this leads to these configurations preserving $(4,0)$ supersymmetry. Turning to the third case of fivebranes wrapping a Kähler four-cycle in a Calabi-Yau four-fold $X$, the projections for $X$ now give

$$
\begin{aligned}
& \Gamma^{1234} \boldsymbol{\epsilon}=\boldsymbol{\epsilon} \\
& \Gamma^{3456} \boldsymbol{\epsilon}=\boldsymbol{\epsilon} \\
& \Gamma^{5678} \boldsymbol{\epsilon}=\boldsymbol{\epsilon},
\end{aligned}
$$


while the presence of the brane implies the same condition (60) as above. This projection is only compatible with the $X$ projections for $\epsilon^{-}$. Thus $Y$ has a single $S U(4)$ complex structure $J^{-}$. Consequently only one chirality of the spinor is preserved and the theory preserves $(2,0)$ supersymmetry in $D=2$. Again the generalised calibration condition (61) is satisfied for $J^{-}$. As in the $S U(3)$ case, these geometries with $S U(4)$ holonomy also have one or two holomorphic four-forms $\mathrm{e}^{-2 \Phi} \omega^{ \pm}$, which are generalised calibrations corresponding to what are special Lagrangian calibrations in case of pure geometry where $H=\Phi=0$.

Eight manifolds $Y$ with $\operatorname{Spin}(7)$ holonomy can arise in three ways. First, consider fivebranes wrapping co-associative four-cycles in $X$ with $G_{2}$ holonomy, a case with one overall transverse direction. The projections for $X$ were given in (50). The brane projection reads

$$
\Gamma^{5678} \boldsymbol{\epsilon}=\tau_{3} \epsilon
$$

The combined projections are then equivalent to two different $\operatorname{Spin}(7)$ structures on $Y$. In particular they can be rewritten as

$$
\begin{aligned}
& \Gamma^{1234} \boldsymbol{\epsilon}=\boldsymbol{\epsilon} \\
& \Gamma^{3456} \boldsymbol{\epsilon}=\boldsymbol{\epsilon} \\
& \Gamma^{5678} \boldsymbol{\epsilon}=\tau_{3} \boldsymbol{\epsilon} \\
& \Gamma^{1357} \boldsymbol{\epsilon}=\boldsymbol{\epsilon} .
\end{aligned}
$$

Again the two structures are related by reversing the overall transverse tangent direction $e^{8}$ and give two different Cayley structures $\Omega^{-}$and $\Omega^{+}$on $Y$ with $\nabla^{-} \Omega^{-}=0$ and $\nabla^{+} \Omega^{+}=0$. Note in particular that the eight-dimensional chirality of $\epsilon^{-}$and $\epsilon^{+}$on $Y$ are different. This means that the theory preserves $(1,1)$ supersymmetry in $D=2$. As before one can derive a generalized calibration condition by taking $A=\gamma_{\alpha_{1} \alpha_{2} \alpha_{3} \alpha_{4} \alpha_{5}}$ in the analog of (55), giving

$$
H=\mathrm{e}^{2 \Phi} *_{8} \mathrm{~d}\left(\mathrm{e}^{-2 \Phi} \Omega^{ \pm}\right) \text {. }
$$

As above the difference in chirality of $\epsilon^{-}$and $\epsilon^{+}$means there is no overall sign factor.

The second way to obtain $Y$ with $\operatorname{Spin}(7)$ holonomy is by wrapping SLAG fourcycles in a Calabi-Yau fourfold $X$ with $S U(4)$ holonomy. The projections for $X$ are given by (64). The brane projection reads

$$
\Gamma^{1357} \boldsymbol{\epsilon}=\tau_{3} \boldsymbol{\epsilon} .
$$

Again this leads to two different $\operatorname{Spin}(7)$ structures $\Omega^{ \pm}$on $Y$. However, in contrast to the last case, these structures have the same eight-dimensional chirality. Thus this theory preserves $(2,0)$ supersymmetry in $D=2$. Note that this is also the first case of $Y$ with a pair of structures when there were no overall transverse directions. As in the previous case, both structures satisfy the generalised calibration condition (67), except since $\epsilon^{-}$and $\epsilon^{+}$have the same chirality there is an overall sign factor

$$
H=\mp \mathrm{e}^{2 \Phi} *_{8} \mathrm{~d}\left(\mathrm{e}^{-2 \Phi} \Omega^{ \pm}\right)
$$


The last case with $\operatorname{Spin}(7)$ holonomy comes from wrapping Cayley cycles in $X$ with $\operatorname{Spin}(7)$ holonomy. The projections from $X$ are

$$
\begin{aligned}
& \Gamma^{1234} \boldsymbol{\epsilon}=\boldsymbol{\epsilon} \\
& \Gamma^{3456} \boldsymbol{\epsilon}=\boldsymbol{\epsilon} \\
& \Gamma^{5678} \boldsymbol{\epsilon}=\boldsymbol{\epsilon} \\
& \Gamma^{1357} \boldsymbol{\epsilon}=\boldsymbol{\epsilon},
\end{aligned}
$$

and the brane gives

$$
\Gamma^{5678} \boldsymbol{\epsilon}=\tau_{3} \boldsymbol{\epsilon} .
$$

This is only consistent with the other projections for $\epsilon^{-}$. Thus there is only one Cayley structure $\Omega^{-}$on $Y$, again satisfying the generalised calibration condition (67). Since only one chirality of spinor survives, the theory preserves $(1,0)$ supersymmetry in $D=2$.

Finally, let us discuss fivebranes wrapping complex Lagrangian four-cycles in hyper-Kähler manifolds. These are four-cycles that are complex with respect to one complex structure and special Lagrangian with respect to the other two. Solutions for M-fivebranes wrapping these cycles were recently given in [17], and we will use the analysis at the beginning of section 2 of that paper. Let the projections corresponding to the $S p(2)$ singlets of a hyper-Kähler manifold $X$ be given by

$$
\begin{array}{r}
\Gamma^{1256} \boldsymbol{\epsilon}=\boldsymbol{\epsilon} \\
\Gamma^{3478} \boldsymbol{\epsilon}=\boldsymbol{\epsilon} \\
\left(1-\Gamma^{1234}-\Gamma^{1458}-\Gamma^{2358}\right) \boldsymbol{\epsilon}=0 .
\end{array}
$$

These preserve $(6,0)$ supersymmetry in $D=2$. One can easily show [17] that the chirality of the three preserved $\epsilon^{-}$spinors with respect to $\Gamma^{1234}, \Gamma^{1458}$ and $\Gamma^{2358}$ can be taken to be $(+,+,-),(+,-,+)$ and $(-,+,+)$, respectively, and similarly for the three preserved $\epsilon^{+}$spinors. The four-cycle tangent to the 1234 directions is complex Lagrangian and the corresponding projections on the spinors can be written

$$
\Gamma^{1234} \boldsymbol{\epsilon}=\tau_{3} \boldsymbol{\epsilon} .
$$

We see that the configuration preserves the first two $\epsilon^{-}$spinors and the third $\epsilon^{+}$spinor, giving $(3,0)$ supersymmetry in $D=2$. The two $\epsilon^{-}$spinors satisfy the same projections as those preserved by a fivebrane wrapping a Kähler four-cycle in a Calabi-Yau fourfold and imply that $\nabla^{-}$has $S U(4)$ holonomy. On the other hand, the single preserved $\epsilon^{+}$spinor implies that $\nabla^{+}$has $\operatorname{Spin}(7)$ holonomy.

We have summarised the results of this section in the following table. We have also included the amount of supersymmetry preserved in type IIA and type I supergravity. For type IIB and type IIA the amount of preserved supersymmetry is the same irrespective of the orientation of the fivebrane. The cases where this is not true for the type I theory are indicated in the last column of the table.

Note that supergravity solutions describing intersecting branes calibrated by quaternionic calibrations were discussed in [39] where the holonomy of the connections $\nabla^{ \pm}$ was also deduced. Note that unlike the cases we have been considering, these quaternionic cycles are necessarily linear 40]. 


\begin{tabular}{|cc|ccc|cccc|}
\hline \multicolumn{2}{|c|}{ Probe configuration } & \multicolumn{4}{|c|}{ Geometry of $Y$} & \multicolumn{4}{c|}{ World volume susy } \\
Cycle $\Sigma$ & $X$ & $\operatorname{dim}(Y)$ & $\operatorname{Hol}\left(\nabla^{-}\right)$ & $\operatorname{Hol}\left(\nabla^{+}\right)$ & $D$ & $\mathcal{N}_{\text {IIB }}$ & $\mathcal{N}_{\text {IIA }}$ & $\mathcal{N}_{\text {I }}$ \\
\hline \hline Kähler-2 & $\mathrm{CY}_{2}$ & 6 & $S U(3)$ & $S U(3)$ & 4 & 8 & 8 & 4 \\
\hline Kähler-2 & $\mathrm{CY}_{3}$ & 6 & $S U(3)$ & $S O(6)$ & 4 & 4 & 4 & 4 or none \\
\hline SLAG-3 & $\mathrm{CY}_{3}$ & 7 & $G_{2}$ & $G_{2}$ & 3 & 4 & 4 & 2 \\
\hline Associative & $G_{2}$ & 7 & $G_{2}$ & $S O(7)$ & 3 & 2 & 2 & 2 or none \\
\hline Kähler-4 & $\mathrm{CY}_{3}$ & 8 & $S U(4)$ & $S U(4)$ & 2 & $(2,2)$ & $(4,0)$ & $(2,0)$ \\
\hline$(\text { Kähler-2 })^{2}$ & $\mathrm{CY}_{2} \times \mathrm{CY}_{2}$ & 8 & $S U(4)$ & $S U(4)$ & 2 & $(4,0)$ & $(2,2)$ & $(2,0)$ \\
\hline CLAG-4 & hyper-Kähler & 8 & $S U(4)$ & $S p i n(7)$ & 2 & $(3,0)$ & $(2,1)$ & $(2,0)$ or $(1,0)$ \\
\hline Kähler-4 & $\mathrm{CY}_{4}$ & 8 & $S U(4)$ & $S O(8)$ & 2 & $(2,0)$ & $(2,0)$ & $(2,0)$ or none \\
\hline SLAG-4 & $\mathrm{CY}_{4}$ & 8 & $S p i n(7)$ & $S p i n(7)$ & 2 & $(2,0)$ & $(1,1)$ & $(1,0)$ \\
\hline Co-associative & $G_{2}$ & 8 & $S p i n(7)$ & $S p i n(7)$ & 2 & $(1,1)$ & $(2,0)$ & $(1,0)$ \\
\hline Cayley & $S p i n(7)$ & 8 & $S p i n(7)$ & $S O(8)$ & 2 & $(1,0)$ & $(1,0)$ & $(1,0)$ or none \\
\hline
\end{tabular}

Table 1: Holonomy and supersymmetry of supergravity solutions for wrapped NS five-branes.

\section{Discussion}

We have presented a $D=10$ supergravity solution describing IIB fivebranes wrapping a SLAG three-cycle and discussed how it can be interpreted as a gravity dual of pure $\mathcal{N}=2$ super-Yang-Mills theory in $D=3$. It would be very interesting to extend this work to find gravity solutions dual to $\mathcal{N}=2$ theories with a Chern-Simons term since these can have supersymmetric confining vacua. A Chern-Simons term arises when there is NS three-form flux on the SLAG three-sphere [5]. In order to construct such solutions within gauged supergravity, one needs to switch on the $D=7$ three-form. However, upon inspection of the equations of motion [28] it is not difficult to see that one has to go beyond the $S O(3)$ ansatz that we considered in this paper.

We have also discussed some aspects of the geometry arising when IIB fivebranes wrap supersymmetric cycles. In particular we argued that in some cases one obtains geometries with both of the connections with torsion, $\nabla^{ \pm}$, having special holonomy and in others only one of them does. For each case we also elucidated the appropriate notion of generalised calibration. Explicit supergravity solutions for several different cases of fivebranes wrapping supersymmetric cycles have now been found. It seems straightforward to find solutions for all cases, by first constructing them in gauged supergravity, and these will provide explicit examples of the remaining geometries that we discussed. It would be interesting to see if the new cases have a dual field theory interpretation. 


\section{Acknowledgements}

We thank Fay Dowker, Jan Gutowski, Chris Hull, and Stathis Pakis for helpful discussions. All authors are supported in part by PPARC through SPG \#613. JPG thanks EPSRC for partial support. DW is also supported by the Royal Society.

\section{References}

[1] M. Bershadsky, C. Vafa and V. Sadov, "D-Branes and Topological Field Theories," Nucl. Phys. B463 (1996) 420, hep-th/9511222.

[2] J. Maldacena and C. Nunez, "Supergravity description of field theories on curved manifolds and a no go theorem," Int. J. Mod. Phys. A 16, 822 (2001) hepth/0007018.

[3] J. M. Maldacena and C. Nunez, "Towards the large $N$ limit of pure $\mathcal{N}=1$ super Yang Mills," Phys. Rev. Lett. 86, 588 (2001) hep-th/0008001.

[4] B. Brinne, A. Fayyazuddin, S. Mukhopadhyay and D. J. Smith, "Supergravity M5-branes wrapped on Riemann surfaces and their QFT duals," JHEP 0012, 013 (2000) hep-th/0009047.

[5] B. S. Acharya, J. P. Gauntlett and N. Kim, "Fivebranes wrapped on associative three-cycles,", to appear in Phys. Rev. D hep-th/0011190.

[6] H. Nieder and Y. Oz, "Supergravity and D-branes wrapping special Lagrangian cycles," JHEP 0103, 008 (2001) hep-th/0011288.

[7] J. P. Gauntlett, N. Kim and D. Waldram, "M-fivebranes wrapped on supersymmetric cycles," Phys. Rev. D 63, 126001 (2001) hep-th/0012195.

[8] C. Nunez, I. Y. Park, M. Schvellinger and T. A. Tran, "Supergravity duals of gauge theories from $F(4)$ gauged supergravity in six dimensions," JHEP 0104, 025 (2001) hep-th/0103080.

[9] J. D. Edelstein and C. Nunez, "D6 branes and M-theory geometrical transitions from gauged supergravity," JHEP 0104, 028 (2001) hep-th/0103167.

[10] M. Schvellinger and T. A. Tran, "Supergravity duals of gauge field theories from $S U(2) \times U(1)$ gauged supergravity in five dimensions," JHEP 0106, 025 (2001) hep-th/0105019.

[11] J. Maldacena and H. Nastase, "The supergravity dual of a theory with dynamical supersymmetry breaking," JHEP 0109, 024 (2001) hep-th/0105049.

[12] J. P. Gauntlett, N. Kim, S. Pakis and D. Waldram, "Membranes wrapped on holomorphic curves," to appear in Phys. Rev. D, hep-th/0105250. 
[13] R. Hernandez, "Branes wrapped on coassociative cycles," hep-th/0106055.

[14] J. P. Gauntlett, N. Kim, D. Martelli and D. Waldram, "Wrapped fivebranes and $\mathcal{N}=2$ super Yang-Mills theory," to appear in Phys. Rev. D, hep-th/0106117.

[15] F. Bigazzi, A. L. Cotrone and A. Zaffaroni, "N $\mathcal{N}=2$ gauge theories from wrapped five-branes," hep-th/0106160.

[16] J. Gomis and T. Mateos, "D6 branes wrapping Kaehler four-cycles," hepth/0108080.

[17] J. P. Gauntlett and N. Kim, "M-fivebranes wrapped on supersymmetric cycles. II," hep-th/0109039.

[18] I. Affleck, J. A. Harvey and E. Witten, "Instantons And (Super)Symmetry Breaking In (2+1)-Dimensions," Nucl. Phys. B 206, 413 (1982).

[19] J. de Boer, K. Hori and Y. Oz, "Dynamics of $\mathcal{N}=2$ supersymmetric gauge theories in three dimensions," Nucl. Phys. B 500, 163 (1997) hep-th/9703100.

[20] O. Aharony, A. Hanany, K. Intriligator, N. Seiberg and M. J. Strassler, "Aspects of $\mathcal{N}=2$ supersymmetric gauge theories in three dimensions," Nucl. Phys. B 499, 67 (1997) hep-th/9703110.

[21] K. Ohta, "Supersymmetric index and s-rule for type IIB branes," JHEP 9910, 006 (1999) hep-th/9908120.

[22] A. H. Chamseddine and M. S. Volkov, "Non-Abelian vacua in $D=5, \mathcal{N}=4$ gauged supergravity," JHEP 0104, 023 (2001) hep-th/0101202.

[23] G. Papadopoulos and A. A. Tseytlin, "Complex geometry of conifolds and 5-brane wrapped on 2-sphere," Class. Quant. Grav. 18, 1333 (2001) hepth/0012034.

[24] J. Gomis and J. G. Russo, " $D=2+1 \mathcal{N}=2$ Yang-Mills Theory From Wrapped Branes," hep-th/0109177.

[25] N. Seiberg, "New theories in six dimensions and matrix description of M-theory on $T^{5}$ and $T^{5} / Z_{2}, "$ Phys. Lett. B 408, 98 (1997) hep-th/9705221.

[26] R. C. McLean, "Deformations of calibrated submanifolds," Comm. Anal. Geom. 6 (1998) 705-747.

[27] A. Salam and E. Sezgin, " $S O(4)$ Gauging Of $\mathcal{N}=2$ Supergravity In SevenDimensions," Phys. Lett. B 126, 295 (1983).

[28] M. Cvetič, H. Lü and C.N. Pope, "Consistent Kaluza-Klein Sphere Reductions," Phys. Rev. D62 (2000) 064028, hep-th/0003286. 
[29] M. Abramowitz and I. A. Stegun (editors), Handbook of mathematical functions, Dover Publications, New York (1965).

[30] A. Buchel, A. W. Peet and J. Polchinski, "Gauge dual and noncommutative extension of an $\mathcal{N}=2$ supergravity solution," Phys. Rev. D 63, 044009 (2001) hep-th/0008076.

[31] N. Itzhaki, J. M. Maldacena, J. Sonnenschein and S. Yankielowicz, "Supergravity and the large $N$ limit of theories with sixteen supercharges," Phys. Rev. D 58 (1998) 046004 hep-th/9802042.

[32] J. Gutowski and G. Papadopoulos, "AdS calibrations," Phys. Lett. B 462 (1999) 81 hep-th/9902034.

[33] J. Gutowski, G. Papadopoulos and P. K. Townsend, "Supersymmetry and generalized calibrations," Phys. Rev. D 60 (1999) 106006 hep-th/9905156.

[34] C. Schmidhuber, "D-brane actions," Nucl. Phys. B 467 (1996) 146 hepth/9601003.

[35] T. Friedrich and S. Ivanov, "Parallel spinors and connections with skewsymmetric torsion in string theory," math.dg/0102142.

[36] A. Strominger, "Superstrings With Torsion," Nucl. Phys. B 274 (1986) 253.

[37] S. J. Gates, C. M. Hull and M. Rocek, "Twisted Multiplets And New Supersymmetric Nonlinear Sigma Models," Nucl. Phys. B 248 (1984) 157.

[38] C. M. Hull, "Superstring Compactifications With Torsion And Space-Time Supersymmetry," In Turin 1985, Proceedings, Superunification and Extra Dimensions, 347-375.

[39] G. Papadopoulos, "Brane solitons and hypercomplex structures," math.dg/0003024.

[40] J. Dadok, F.R. Harvey and F. Morgan, "Calibrations on $R^{8}$," Trans. Am. Math. Soc. 307 (1988) 1. 